\title{
Determination of Thin-Film Ferroelectric Polarity at the Nanoscale
}

\author{
J. M. LeBeau,* A. J. D’Alfonso, ${ }^{* *}$ L. J. Allen, ${ }^{* *}$ and S. Stemmer*** \\ * $\quad$ Materials Science \& Engineering, North Carolina State University, Raleigh, NC 27606 \\ ** School of Physics, University of Melbourne, Victoria 3010, Australia \\ *** Materials Department, University of California, Santa Barbara, CA 93106-5050
}

The properties of thin-film ferroelectric materials depend on the spatial arrangement (domain patterns) of the ferroelectric polarization. Transmission electron microscopy has been used successfully for the analysis of specimen polarity of bulk materials [1] and thin films [2]; however, determining the direction of polarity (such as across $180^{\circ}$ domain boundaries) is challenging when unit cell distortions are small, such as in $\mathrm{BaTiO}_{3}$. And while convergent beam electron diffraction (CBED) can distinguish between $90^{\circ}$ and $180^{\circ}$ domain walls [3], typical beam size on the order of 10 $\mathrm{nm}$ limits utility for examining nanoscale variations of the ferroelectric polarization in thin-film materials.

In this presentation, we establish that position averaged convergent beam electron diffraction (PACBED) allows for determination of the polarity in ferroelectric materials. The PACBED technique uses a scanned Ångström-sized electron probe in STEM to form patterns that exhibit high sensitivity to specimen thickness, tilt, and crystal structure polarity [4]. We will demonstrate that PACBED allows for ferroelectric polarity determination even when unit-cell distortions are smaller than what can be measured using real-space imaging techniques. The sensitivity of the technique is demonstrated by examining a thin-film of $\mathrm{BaTiO}_{3}$ grown by molecular beam epitaxy on $\mathrm{SrTiO}_{3}$ where the polar distortion is only $\sim 4 \mathrm{pm}$. Further, we will show that the method is compatible with the electron optical settings and specimen thicknesses used in atomic resolution STEM imaging.

Fig. 1 shows a simulated polar $\mathrm{BaTiO}_{3}$ PACBED pattern. The pattern exhibits asymmetric intensity, particularly in the lobes at the top and bottom of the central disk, due to non-centrosymmetricity of the unit-cell (see figure for orientation). Fig. 2 shows magnified top and bottom lobes as a function of specimen thickness where asymmetry is detectable by visual inspection over a range of specimen thicknesses. Validation of the technique is provided in Fig. 3 where excellent agreement is demonstrated between an experimental PACBED pattern of a $\mathrm{BaTiO}_{3}$ thin-film, acquired from a $\sim 5$ $\times 5$ unit-cell region, and a corresponding simulation. We will also show that the $a$ and $c$ directions of a tetragonal unit cell can also be distinguished. When this information is combined with the asymmetric lobe intensity, separation of unit-cell distortions and polarity is made possible. For example, cases where a tetragonal film may not necessarily be polar can be investigated with PACBED patterns. Finally, we will also address the influence of specimen tilt on the PACBED intensity distribution for polar materials.

References

[1] M. Tanaka and G. Honjo, Journal of the Physical Society of Japan. 19 (1964), 954.

[2] C. L. Jia, et al., Nature Materials. 6 (2007), 64.

[3] Z. H. Zhang, X. Y. Qi, and X. F. Duan, Applied Physics Letters. 89 (2006), 242905.

[4] J. M. LeBeau, S. D. Findlay, L. J. Allen, and S. Stemmer, Ultramicroscopy, 110 (2010), 118. 

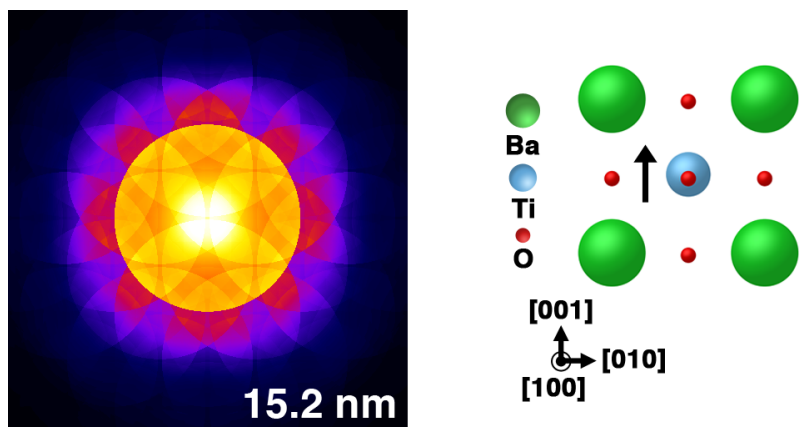

FIG. 1. Simulated PACBED pattern of $\mathrm{BaTiO}_{3}$ with corresponding orientation of the unit-cell.
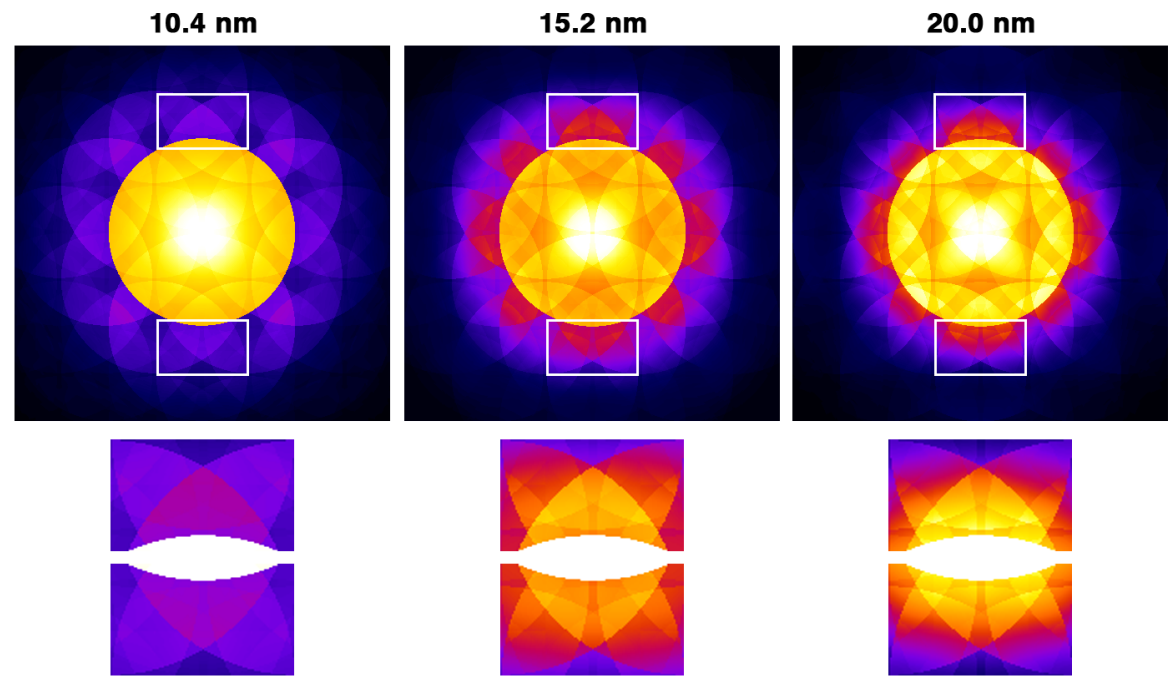

FIG. 2. (top) Simulated PACBED patterns of $\mathrm{BaTiO}_{3}$ as function of thickness (bottom) Magnified lobes of the PACBED patterns, as indicated, where image intensities have been adjusted to accentuate the lobe asymmetry.

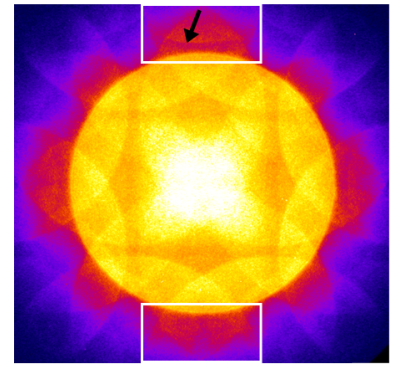

Experiment

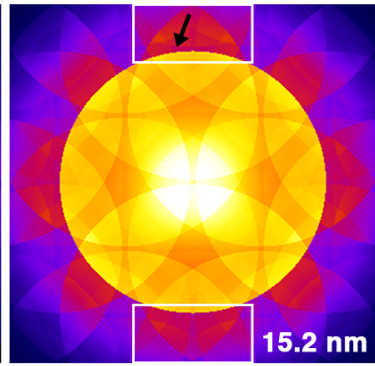

Simulation
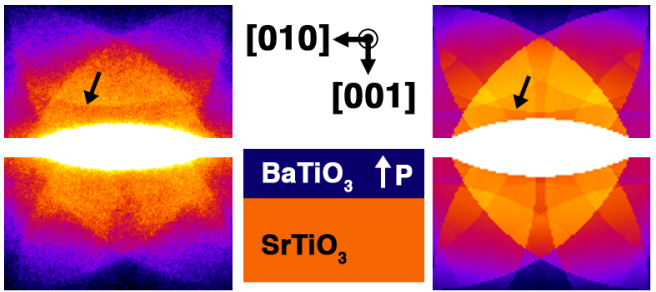

FIG. 3. (left) Experimental and simulated PACBED patterns of a $15.2 \mathrm{~nm}$ thick $\mathrm{BaTiO}_{3}$ thin-film sample (right) Comparison of the top and bottom lobes extracted from experiment and simulations. Inset shows the orientation of the $\mathrm{BaTiO}_{3}$ thin-film ferroelectric polarization relative to the substrate. 